\title{
LOADING DRIVERS TO THEIR LIMIT: THE EFFECT OF INCREASING SECONDARY TASK ON DRIVING
}

\author{
Natasha Merat \\ Institute for Transport Studies \\ University of Leeds \\ Leeds LS2 9JT \\ UK \\ E-mail: nmerat@its.leeds.ac.uk
}

\begin{abstract}
Summary: This paper outlines the results of a number of studies that were designed to create a series of suitable "secondary tasks" that would act as appropriate surrogate in-vehicle information systems (s-IVIS). In particular, an attempt was made to design tasks that would allow a step-by-step increase in visual or cognitive load. Following a brief introduction to the development of each s-IVIS, the paper will present results from a study which examined the effect of completing an auditory s-IVIS on a simulated driving task.
\end{abstract}

\section{INTRODUCTION}

In-vehicle information systems (IVIS) encompass a variety of on-board devices, which are collectively designed to present an assortment of information to the driver. These devices may function as navigation or traffic information systems, as well as driver monitoring systems offering feedback on driving performance. Typically, the degree of visual and/or cognitive load imposed by these systems can vary substantially during a drive, and the effect of this workload on driving performance and safety can depend on a series of factors such as road condition, traffic density and driver ability.

As shown in Figure 1, safe driving and successful IVIS operation can be influenced by an interaction between driver characteristics, IVIS type and road conditions. Therefore, from a methodological viewpoint, it is important to establish how the type of information presented by an IVIS and the complexities associated with this information influence driving performance, and if this

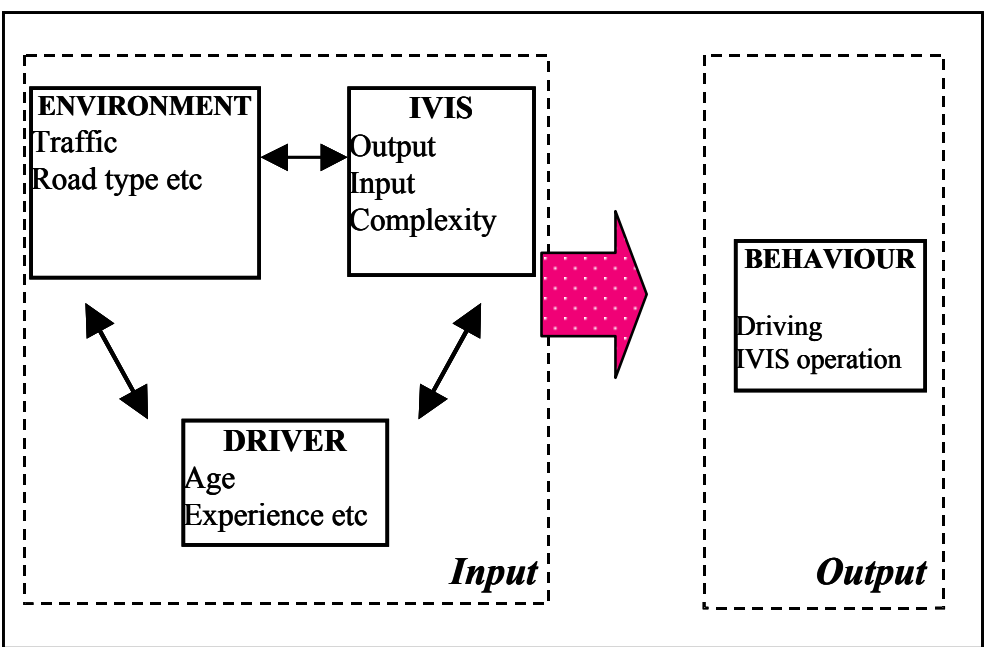

Figure 1. The interaction between Driver-Environment-IVIS interaction varies between traffic and road conditions.

The study of such interactions is one of the main aims of the EU-funded project HASTE (Human

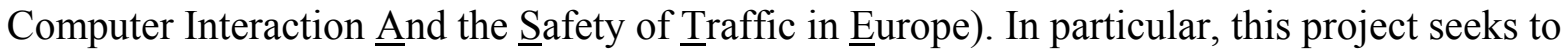


examine how a systematic increase in processing load imposed by an IVIS, affects driving performance. In addition, the effect of IVIS presentation rate, presentation mode and response method on driving in different scenarios will be examined. The results of this research are intended to assist in the safety assessment of in-vehicle devices.

The first stage of the HASTE project involved developing a series of PC-based laboratory tasks, based on the following objectives:

- To create tasks which as much as possible would impose either a visual load or a cognitive load

- To develop tasks which would bring about a systematic increase in workload, as indicated by an increase in the number of errors and/or reaction time

- To study the effect of rate of presentation, number of items presented, mode of presentation and mode of response on single task performance

The above objectives were used to conduct a series of pilot studies. Following these pilot studies, a visual search task and an auditory memory task were selected for use as 'surrogate' IVIS, to study the interaction between increasing secondary task difficulty and driving. For the purpose of this paper, the development of the cognitively loading auditory memory task will be described.

\section{EXPERIMENT 1}

\section{Method}

The auditory continuous memory task (aCMT) was designed to study the effect of a cognitively loading non-visual task on driving. Adapted from the visual version which used letters of the alphabet (Veltman \& Gaillard, 1998), this task requires subjects to maintain a count of their 'target sound,' which is presented within a sequence of non-target sounds.

Two PC-based between-subject experiments were conducted. For both experiments, each target sound was presented a maximum of three times within a block, and a maximum of four sounds were presented within any one sequence. Crucially, when more than one target sound was presented, subjects were required to maintain a separate tally for each target sound.

The sound samples were chosen after a series of pilot studies and fulfilled the following criteria:

- Intensity was normalised across all sounds

- All sound samples chosen were judged easily distinguishable from each other

- Average sound duration was $160 \mathrm{~ms}( \pm 28 \mathrm{~ms})$

Results from the visual version of the task have shown an increase in error with an increase in the number of target letters to be remembered. For the auditory version of the task, we studied the effect of list length, number of target sounds, rate of presentation and response mode on performance. These manipulations are summarized in Table 1. 
Table 1. The list of variables used Experiments $1 \mathrm{~A}$ and $1 \mathrm{~B}$

\begin{tabular}{lllll}
\hline Experiment & List Length & Rate of presentation & Response mode & $\mathrm{N}$ \\
\hline 1A & 10,20 & Every second & Manual & 36 \\
1B & 15 & Every 2 secs, Every 5 secs & Manual, Verbal & 12 \\
\hline
\end{tabular}

\section{Results}

For each experiment, a repeated measures ANOVA was conducted on the percentage of correct responses, percentage incorrect answers (when the incorrect count was given), percentage of false positives (when response to a non-target sound was given) and percentage of missed answers.

Results from Experiment $1 \mathrm{~A}$ failed to reveal an effect of list length on accuracy $(\mathrm{F}(1,35)=.73$, $\mathrm{p}=.4)$. Also, in Experiment $1 \mathrm{~B}$, accuracy was not found to be influenced by mode of response ( $\mathrm{F}$ $(1,11)=.004, p=.952)$ or rate of presentation $(\mathrm{F}(1,11)=.03, \mathrm{p}=.866)$. However, the ANOVA did reveal a reliable interaction between rate of presentation and number of target sounds, $(\mathrm{F}$ $(2,22)=7.265, \mathrm{p}<.05)$. Post hoc tests revealed a higher percentage accuracy for the two target sound condition when sounds were presented at the faster rate of every two seconds $(p<.05)$.

When sounds were presented at a rate of 1 every second or one every 2 seconds, a reliable fall in percentage accuracy was found with an increase in the number of target sounds $(\mathrm{F}(3,135)=121$, $p<.001$ and $(\mathrm{F}(2,22)=11.79, p<$ .01 respectively). However, this systematic fall in accuracy was not seen when sounds were presented every 5 seconds (see Figure 2). Finally, increasing the number of target sounds was also found to increase the percentage of incorrect answers, false positives, and missed answers.

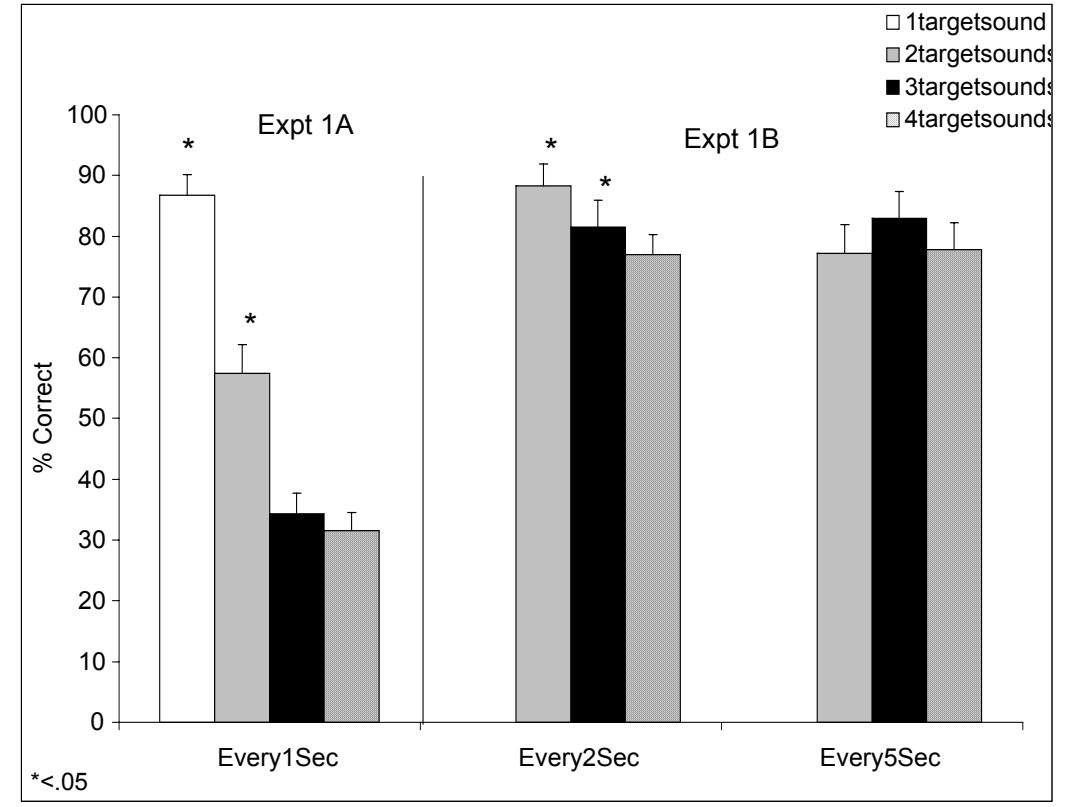

Figure 2 - Performance in the aCMT task 


\section{Discussion}

The main aim of the above experiments was to establish the most suitable criteria to be used in assessing the effect of increasing cognitive load on a driving task within the scope of the HASTE project. Based on the above results, we can conclude that the auditory continuous memory task can be used to assess the effect of a systematic increase in cognitive load on driving. While analyses failed to show a reliable effect of presentation rate on performance accuracy, the number of missed responses in the fast presentation rate condition (every second) and the mixed pattern of response achieved in the slow presentation condition (every 5 seconds) encouraged the use of the medium rate of every two seconds for future studies. Also, since mode of presentation was not found to have an effect on accuracy, a verbal mode of response was selected for use in the driving studies, to reduce the amount of conflict between manual responses to the auditory task and driving. Finally, since no reliable differences in accuracy were found between the two different list lengths used in Experiment 1A, we decided upon the use 15 sounds per block for the next stage of experiments.

\section{EXPERIMENT 2}

The main aim of this experiment was to assess the interaction between aCMT and driving, using a variety of road types and scenarios. However, for the purpose of this paper, the effect of aCMT on driving in a rural road environment will be outlined.

\section{Road Environment}

Two rural experimental routes were created. Each road was 3.65 metres wide and just over $22 \mathrm{~km}$ long, with 'filler' sections separating the experimental portions of each route. A lead car was introduced at the beginning of each route, and followed a controlled speed and headway profile throughout the drive. This allowed a 'car following' task in designated sections of each road coinciding with aCMT presentation, and 'free driving' in other sections. Car following was introduced to maximize data collection in driving performance during secondary task presentation.

The three levels of driving complexity were each repeated three times within both roads, producing a 3 (road complexity) x 3 (aCMT level) repeated measures design. The three levels of road complexity were: (i) a straight section (ii) an s-shaped curve section and (iii) a 'discrete event' on an s-shaped curve section. The layout of the two routes was exactly the same, but the discrete events were changed between the two routes to reduce monotony. All six discrete events (three for each route) involved a smooth deceleration of the lead car due to the presence of other objects or cars on the road. 


\section{Participants and Design}

Twelve participants ( 5 male, 7 female) took part in this study. All participants completed a practice session of the aCMT task, and a practice drive. The two routes and a baseline version of the aCMT were then completed by all participants, in a counter-balanced order. Half of the subjects drove Route 1 in combination with the aCMT, and the other half completed the aCMT during their drive of Route 2. The aCMT was presented nine times during a particular drive, and each block lasted just over 30 seconds. The difficulty level of the blocks was counterbalanced across subjects.

\section{Results}

\section{Auditory Continuous}

Memory task

The percentage of correct responses achieved for aCMT during the baseline and driving conditions were subjected to a 3 (number of target sounds) $\mathrm{x} 4$ (no driving, straight road, sshaped curve, discrete event) repeated measure analysis of variance. The main effect of increasing target sounds was found to be reliable $(\mathrm{F}(2,22)$ $=3.72, p<.01$, see Figure 3).

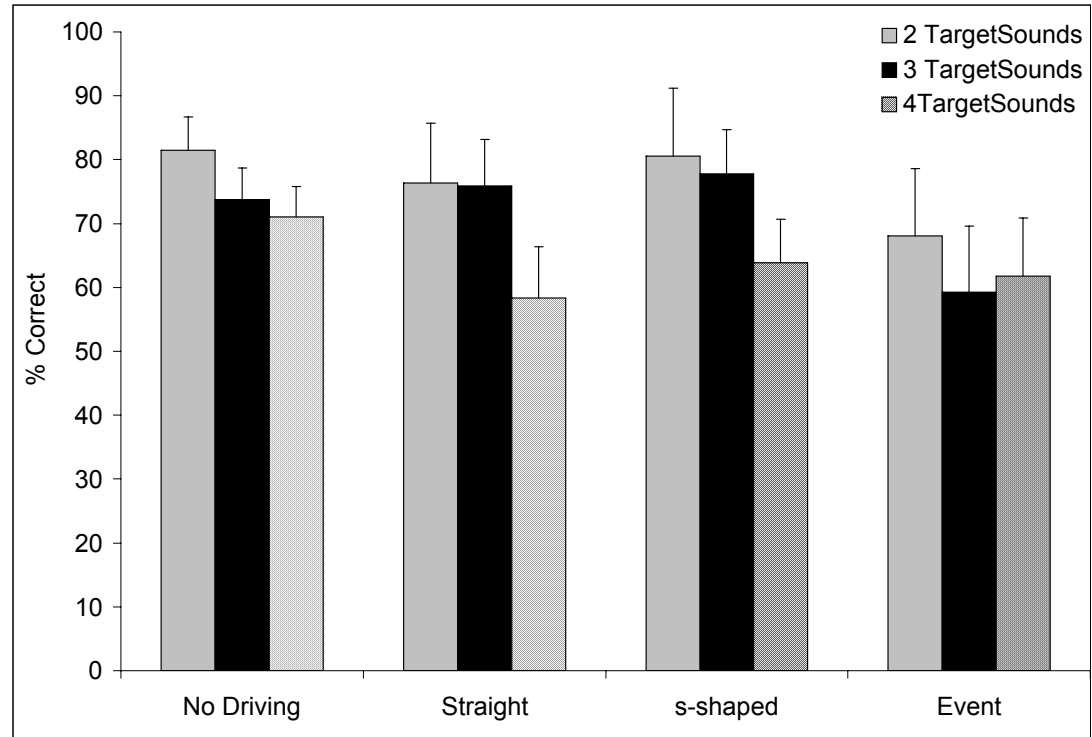

Figure 3. Performance in the aCMT during driving

Post-hoc comparisons revealed that the mean percentage of correct answers for the four target sound condition was reliably lower than that of the two target sound condition, during both single and dual task performance. Results failed to show an effect of driving on performance in the aCMT, although performance was worst in the 'discrete events' condition.

A range of performance measures were recorded and analysed for the driving task. A summary of the most interesting results will be reported here. A 3 (road complexity) x 4 (aCMT level) analysis of variance showed a reliable reduction in minimum time headway for the discrete event, when compared to baseline $(F(6,66)=4, p<.05$, see Figure 4). Results also revealed a reliable effect of aCMT on minimum time to collision in the discrete event condition (Figure 5), although increasing the difficulty of the task did not affect this measure. 


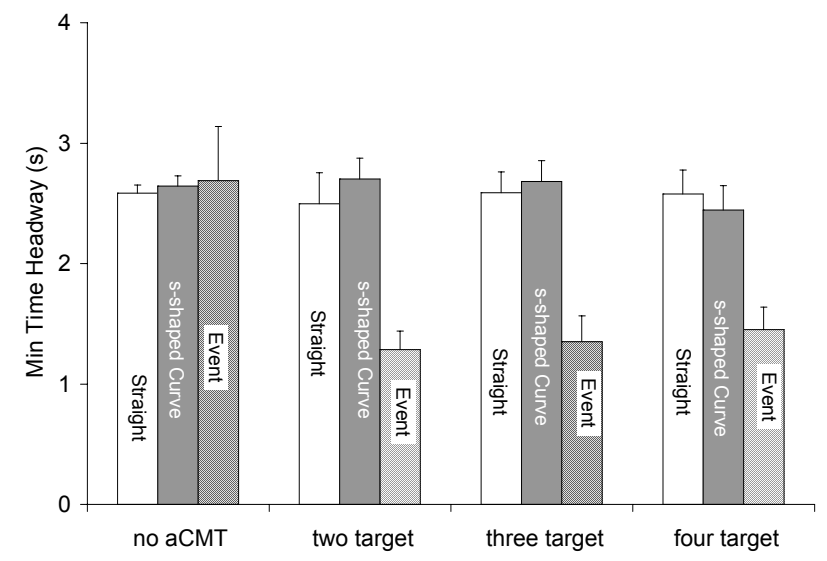

Figure 4. The effect of aCMT on min time headway

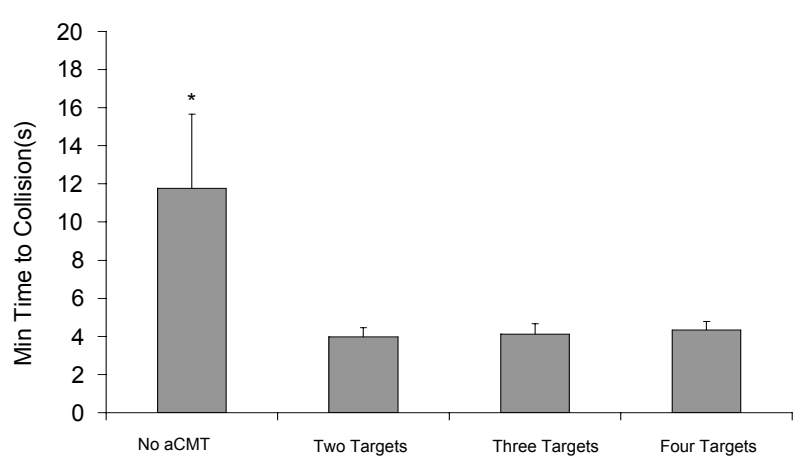

Figure 5. The effect of aCMT on TTC

\section{CONCLUSION}

The experiments described in this paper were designed to study the effect of a systematic increase in 'secondary task' workload on safety in driving. Initial results show an effect of the secondary task on safety in conditions that required drivers to interact with other road users. However, increasing the processing demand of the secondary task was not found to influence driving performance in a systematic fashion. Therefore, at this stage, it may be concluded that the cognitive load imposed by the aCMT was not at a high enough level to impair driving performance in a systematic fashion. Further research is required to address this question.

\section{ACKNOWLEDGMENTS}

The work reported here is supported by the EU-funded project HASTE (GRD1/2000/25361 S12.319626). The author gratefully acknowledges the contribution of Hamish Jamson and Tony Horrobin to the preparation of the simulated driving environment.

\section{REFERENCES}

Veltman, J.A. \& Gaillard, A.W.K. (1998). Physiological workload reactions to increasing levels of task difficulty. Ergonomics, 41(5), 656-669. 